\title{
Adherence to inhaled therapy, mortality and hospital admission in COPD
}

\author{
J Vestbo, ${ }^{1,2} \mathrm{~J}$ A Anderson, ${ }^{3}$ P M A Calverley, ${ }^{4}$ B Celli, ${ }^{5}$ G T Ferguson, ${ }^{6}$ C Jenkins, ${ }^{7}$ \\ K Knobil, ${ }^{8}$ L R Willits, ${ }^{3} \mathrm{~J}$ C Yates, ${ }^{8}$ P W Jones ${ }^{9}$
}

\section{See Editorial, p 922}

- Additional information is published online only at http:// thorax.bmj.com/content/vol64/ issue11

${ }^{1}$ Respiratory Medicine Research Group, University of

Manchester, Manchester, UK;

${ }^{2}$ Department of Cardiology and Respiratory Medicine, Hvidovre Hospital and Faculty of Health Sciences, University of

Copenhagen, Hvidovre Hospital, Hvidovre, Denmark; ${ }^{3}$ Respiratory

Medicine Development Centre,

GlaxoSmithKline, Middlesex, UK;

${ }^{4}$ Clinical Science Centre

University Hospital Aintree,

Liverpool, UK; ${ }^{5}$ Tufts University

School of Medicine, Boston,

Massachusetts, USA;

${ }^{6}$ Pulmonary Research Institute of Southeast Michigan,

Michigan, USA; ${ }^{7}$ Woolcock Institute of Medical Research,

Sydney, Australia; ${ }^{8}$ Respiratory

Medicine Development Centre,

GlaxoSmithKline, Research

Triangle Park, North Carolina,

USA; ${ }^{9}$ St George's, University of

London, London, UK

Correspondence to:

Professor J Vestbo, Respiratory

Medicine Research Group, ERC

Building, 2nd Floor, University

Hospital of South Manchester

NHS Foundation Trust,

Southmoor Road, Manchester

M23 9LT, UK; jorgen.vestbo@

manchester.ac.uk

Received 13 January 2009

Accepted 22 July 2009

Published Online First

20 August 2009

\section{ABSTRACT}

Background: Little is known about adherence to inhaled medication in chronic obstructive pulmonary disease (COPD) and the impact on mortality and morbidity.

Methods: Data on drug adherence from a randomised double-blind trial comparing inhaled salmeterol $50 \mu \mathrm{g}+$ fluticasone propionate $500 \mu \mathrm{g}$ twice daily with placebo and each drug individually in 6112 patients with moderate to severe COPD over 3 years in the TORCH study were used. All-cause mortality and exacerbations leading to hospital admission were primary and secondary end points. The study of adherence was not specified a priori as an ancillary study.

Results: Of the 4880 patients (79.8\%) with good adherence defined as $>80 \%$ use of study medication, $11.3 \%$ died compared with $26.4 \%$ of the 1232 patients (20.2\%) with poor adherence. The annual rates of hospital admission for exacerbations were 0.15 and 0.27 , respectively. The association between adherence and mortality remained unchanged and statistically significant after adjusting for other factors related to prognosis (hazard ratio 0.40 (95\% Cl 0.35 to 0.46$), p<0.001)$. The association was even stronger when analysing ontreatment deaths only. Similarly, the association between adherence and hospital admission remained unchanged and significant in a multivariate analysis (rate ratio 0.58 (95\% Cl 0.44 to $0.73, p<0.001)$. The association between increased adherence and improved mortality and reduction in hospital admission was independent of study treatment. The effect of treatment was more pronounced in patients with good adherence than in those with poor adherence.

Conclusion: Adherence to inhaled medication is significantly associated with reduced risk of death and admission to hospital due to exacerbations in COPD. Further research is needed to understand these strong associations.

Adherence to drug therapy has been shown to be associated with better vital prognosis in a large number of randomised controlled trials. The effect is not trivial and seems independent of the treatment given-that is, the beneficial effect of adherence is similar in patients receiving active treatment with a drug with positive effects to that in patients receiving placebo. ${ }^{1}$ Most of the studies on adherence have used data from trials of cardiovascular treatment as this area contains many large studies with mortality as the main effect parameter. In most of these studies active treatments have had little immediate symptomatic effect or other beneficial effects that could easily be perceived by patients.
Few studies of the effects of adherence to inhaled medication in chronic obstructive pulmonary disease (COPD) have been published and, in general, less is known about adherence in COPD than in asthma. ${ }^{2}$ In a study of elderly subjects including patients with asthma and COPD, good adherence reduced both hospital admissions and physician visits. ${ }^{3}$ Most of the medications used for maintenance treatment of COPD have an immediate symptomatic effect which is easily perceived by patients. ${ }^{4}$ For this reason, drug adherence in COPD is often assumed to be good and associated with disease severity, making patients with severe disease more likely to be adherent to treatment than patients with mild or moderate disease. Nevertheless, very few data are available to support these beliefs. ${ }^{5}$

The Towards a Revolution in COPD Health (TORCH) study was a randomised study which compared salmeterol (SAL), fluticasone propionate (FP), the combination of SAL and FP (SFC) and placebo with overall mortality as the primary end point. ${ }^{6}$ The main findings of the study, which lasted 3 years and included 6112 patients with moderate to severe COPD, have been published elsewhere. ${ }^{7}$ We hypothesised that adherence to inhaled therapy would have a positive impact on mortality and morbidity in COPD and tested the hypothesis using the TORCH study database.

\section{METHODS}

\section{Patients}

Patients with a clinical history of COPD who were current or former smokers with a smoking history of at least 10 pack years, aged 40-80 years, a prebronchodilator forced expiratory volume in $1 \mathrm{~s}$ to forced vital capacity ( $\mathrm{FEV}_{1} / \mathrm{FVC}$ ) ratio $\leqslant 70 \%$ and $\mathrm{FEV}_{1}<60 \%$ of the predicted value, and reversibility of $\mathrm{FEV}_{1}$ to $400 \mu \mathrm{g}$ salbutamol of $<10 \%$ of the predicted value were recruited. The principal exclusion criteria were a diagnosis of asthma or respiratory disorder other than COPD, or an exacerbation during the run-in period.

\section{Study design}

TORCH was a multicentre, randomised, doubleblind, parallel group, placebo controlled study After a 2-week run-in period, eligible patients were stratified by smoking status and randomised to receive either SAL $50 \mu \mathrm{g}$, FP $500 \mu \mathrm{g}$, SFC 50/500 $\mu \mathrm{g}$ or placebo twice daily for 3 years via a Diskus/ Accuhaler inhaler (GlaxoSmithKline, UK). All corticosteroids and inhaled long-acting bronchodilators were stopped before the run-in period, but 
the following COPD medications were allowed: short-acting $\beta_{2}$ agonists as occasional or regular therapy, ipratropium as occasional or regular therapy, regular ipratropium and salbutamol as a combined inhaler and theophylline. The primary efficacy end point of TORCH was all-cause mortality at 3 years. The full methodology has been reported elsewhere. ${ }^{6}$ The study is registered on the publicly accessible database www.clinicaltrials.gov (NCT00268216), GSK study number SCO30003.

\section{Drug adherence}

Study inhalers were collected at study visits every 12 weeks. Patients were supposed to bring all inhalers at these visits and have them exchanged for new ones. Some patients did not adhere to this rule and were then asked to bring the inhalers back at subsequent visits. Inhalers were eventually retrieved but this irregularity in returning inhalers has made it impossible to examine whether adherence changed over time; the Lung Health Study found a gradual decline in adherence over time. ${ }^{8}$ Study drug adherence was evaluated using a dose counter on the device which counted doses of medication left in the Diskus/ Accuhaler inhaler. Patients were not told that we had a particular interest in adherence and no other action likely to encourage patients to simulate use of drugs by emptying the inhalers ("dumping") prior to clinic visits was taken. Good adherence was defined as an average adherence to study medications of $>80 \%$ over the whole period the subject was in the study; poor adherence was defined as $\leqslant 80 \%$. Adherence to concurrent medication was not evaluated. Patients withdrawn from the study were asked to come for a follow-up visit 2 weeks after withdrawal.

\section{Outcome measurements}

The vital status of study patients was followed for 3 years after the start of treatment, regardless of whether they remained on study medication. The primary study end point was time to death from any cause at 3 years after the start of treatment. An independent Clinical Endpoint Committee (CEC) determined the primary cause of death and whether it was COPD-related. The CEC used information from investigators, patient records and other data as available, and was blinded to treatment allocation; the methodology has been reported elsewhere. ${ }^{9}$ Severe exacerbations were defined as exacerbations requiring hospital admission; they were used as a secondary outcome in both the original study and in this analysis. Exacerbation data were only available for those who remained in the study.

\section{Statistical analyses}

The analysis of the effect of adherence on mortality was not specified in the statistical analysis plan or the study protocol of TORCH. The difference in all-cause mortality between patients with good and poor adherence was analysed by the Cox proportional hazards model using time to death as the outcome variable, good adherence as the explanatory variable, and covariates of smoking status, age, gender, region, baseline $\mathrm{FEV}_{1}$, body mass index (BMI), MRC dyspnoea score, previous use of inhaled corticosteroids, previous myocardial infarction and treatment. Interactions between treatment and covariates were tested using this model. Severe exacerbation rates were analysed using a generalised linear model (assuming negative binomial distribution) with number of exacerbations as the outcome. The model included adjustment for effects of the same covariates as for mortality with the addition of number of exacerbations reported in the 12 months prior to screening. In addition, a Cox proportional hazards model using time to first severe exacerbation as the outcome variable was used including the same covariates.

\section{RESULTS}

Of 8554 patients recruited, 6112 comprised the efficacy population; 1524 subjects were allocated to placebo, 1521 to SAL, 1534 to FP and 1533 to SFC. As documented in the primary study report, the treatment groups were similar at baseline. Overall, $79.8 \%$ of the study population had good adherence while in the study. Of the $20.2 \%$ with poor adherence, $61 \%$ (12.3\% of all patients) had $<70 \%$ adherence and only $40 \%(8.0 \%$ of all patients) had $<60 \%$ adherence; $80.3 \%$ of men had good adherence compared with $78.5 \%$ of women. There was no association between GOLD stage and adherence; good adherence was seen in $81 \%, 80 \%$ and $77 \%$ of patients with moderate, severe and very severe COPD, respectively. There was little difference in adherence between treatment groups. Table 1 shows the baseline characteristics for patients with good and poor adherence for each of the four treatment groups. Compared with those with good adherence, patients with poor adherence differed slightly in a number of parameters. Most notably, patients with poor adherence had lower $\mathrm{FEV}_{1}$ at baseline and had more dyspnoea evaluated as confirmation of one the statements "I stop for breath after walking about 100 yards or after a few minutes on the level" or "I am too breathless to leave the house or I am breathless when dressing or undressing" (ie, MRC dyspnoea score 4 and 5).

The overall 3 -year mortality in the study was $14.3 \%$. Of the 4880 patients $(79.8 \%$ ) with good adherence defined as $>80 \%$ use of study medication, $11.3 \%$ died compared with $26.4 \%$ of the 1232 patients $(20.2 \%)$ with poor adherence. The 3-year mortality in groups adherent to SAL, FP, SFC and placebo was $10.7 \%, 12.9 \%, 9.5 \%$ and $12.0 \%$, respectively. In patients with poor adherence, mortality was $25.2 \%, 28.7 \%, 24.9 \%$ and $26.7 \%$, respectively. After adjusting for region, age, sex, smoking status, $\mathrm{FEV}_{1}$, BMI, MRC dyspnoea score, previous use of inhaled corticosteroids, previous myocardial infarction and treatment in a multivariate Cox model, good adherence was associated with a decreased risk of death at any time in the 3-year study period of $60 \%$ (hazard ratio (HR) 0.40 (95\% confidence interval (CI) 0.35 to 0.46$), p<0.001$ ), independent of study treatment. In a model which also included health status assessed using the St George's Respiratory Questionnaire (SGRQ), the HR was 0.41 (95\% CI 0.35 to 0.48 ); this analysis only included 4678 subjects as the SGRQ was not translated into all languages. A KaplanMeier plot of overall mortality for patients with good and poor adherence is shown in fig 1. Kaplan-Meier plots for each treatment group are shown in the online supplement. The Cox model was repeated for deaths while on treatment only. Although this reduced the number of deaths by $47 \%$ to 464 deaths for analysis, the association between adherence and mortality was even stronger (HR 0.25 (95\% CI 0.20 to 0.30$)$ ). Using other cut-off values than $80 \%$ for adherence revealed a clear association between rate of adherence and mortality (data not shown) - the lower the adherence, the higher the mortality. Causes of death are shown in table 2 . The association between adherence and mortality appeared to be independent of the cause of death.

The overall rate of severe exacerbations requiring hospital admission was 0.17 per patient per year. The annual rates of hospitalisation for exacerbations were 0.15 and 0.27 in patients adherent and non-adherent to study medication, respectively. The 3-year admission rates in groups adherent to SAL, FP, SFC 
Table 1 Demographic and baseline characteristics of all patients randomised to treatment with salmeterol (SAL), fluticasone propionate (FP), salmeterol/fluticasone propionate combination (SFC) or placebo according to degree of adherence

\begin{tabular}{|c|c|c|c|c|c|c|c|c|}
\hline & \multicolumn{2}{|c|}{ SAL (N = 1521) } & \multicolumn{2}{|c|}{$\mathrm{FP}(\mathrm{N}=1534)$} & \multicolumn{2}{|c|}{ SFC (N = 1533) } & \multicolumn{2}{|c|}{ Placebo (N = 1524) } \\
\hline & Poor & Good & Poor & Good & Poor & Good & Poor & Good \\
\hline N (\%) & $294(19.3)$ & $1227(80.7)$ & $303(19.8)$ & $1231(80.2)$ & $309(20.2)$ & $1224(79.8)$ & $326(21.4)$ & $1198(78.6)$ \\
\hline Male gender (\%) & 73 & 77 & 76 & 75 & 74 & 75 & 73 & 77 \\
\hline $\mathrm{BMI}\left(\mathrm{kg} / \mathrm{m}^{2}\right)$ & 25.5 & 25.3 & 25.4 & 25.3 & 25.2 & 25.4 & 25.3 & 25.5 \\
\hline Current smoker (\%) & 44 & 43 & 44 & 43 & 44 & 43 & 48 & 42 \\
\hline $\mathrm{FEV}_{1}(\%$ predicted $)$ & 42.4 & 44.1 & 44.2 & 44.7 & 44.3 & 44.8 & 43.6 & 44.4 \\
\hline MRC dyspnoea score 4 and $5(\%)$ & 27 & 17 & 23 & 17 & 22 & 17 & 20 & 18 \\
\hline SGRO total score & 52.1 & 49.3 & 49.4 & 49.6 & 50.1 & 48.5 & 50.8 & 48.5 \\
\hline $\begin{array}{l}\text { Treatment with ICS in year before } \\
\text { study }(\%)^{*}\end{array}$ & 40 & 47 & 45 & 48 & 44 & 50 & 56 & 52 \\
\hline $\begin{array}{l}>1 \text { moderate or severe } \\
\text { exacerbation in year before study } \\
(\%)^{*}\end{array}$ & 33 & 33 & 33 & 32 & 32 & 32 & 36 & 31 \\
\hline
\end{tabular}

Data are mean values unless otherwise indicated.

BMI, body mass index; FEV 1 , forced expiratory volume in 1 s; ICS, inhaled corticosteroid; MRC, Medical Research Council; SGR0, St George Respiratory Questionnaire ${ }^{*}$ Self-reported in the 12 months before screening.

and placebo were $0.14,0.15,0.14$, and 0.16 per patient per year, respectively. In patients with poor adherence, admission rates were $0.26,0.26,0.25$, and 0.36 per patient per year, respectively. After adjusting for region, age, sex, smoking status, $\mathrm{FEV}_{1}, \mathrm{BMI}$, prior exacerbations and treatment in a multivariate model, good adherence was associated with a $44 \%$ lower rate of severe exacerbations (rate ratio 0.56 (95\% CI 0.48 to 0.65 ), $\mathrm{p}<0.001$ ), independent of study treatment. In a model including the SGRQ the rate ratio was 0.54 and, in a Cox regression model with time to first severe exacerbation as outcome and with the same covariates, the HR was 0.59 (95\% CI 0.52 to 0.67 ).

The impact of adherence on the effect of the study drug was evaluated by looking at the primary aim of the TORCH study (ie, to compare the all-cause mortality rate in the groups randomised to placebo and SFC). The absolute risk reduction for SFC compared with placebo was $1.77 \%$ in the group with poor adherence and $2.54 \%$ in the group with good adherence. The relative risk reduction was $6.6 \%$ in the group with poor adherence and $21.2 \%$ in the group with good adherence.

\section{DISCUSSION}

In the TORCH study we observed considerable differences in survival and risk of severe exacerbations according to the degree of adherence with the study drug. Overall, adherence rates were high, and much higher than are usually reported in long-term clinical trials. ${ }^{10}$ Nevertheless, our choice of $80 \%$ adherence as the cut-off value for defining poor adherence is similar to that used in earlier studies of adherence such as those in the cardiovascular area. ${ }^{1}$ The association between increased adherence and improved survival was significant and independent of study treatment.

Findings in line with these have been shown in other therapeutic areas. ${ }^{1}$ We did not have information on adherence to concomitant medications. Patients with COPD often have comorbidities, ${ }^{11}{ }^{12}$ and although it is likely that adherence to study medication also reflects adherence to treatments for comorbidities, it seems improbable that the association between adherence and mortality is merely the effect of better treatment of other coexisting chronic disorders. However, it is possible that patients with comorbidities have poor adherence as a result of treatment with multiple medications and, as they have a poorer prognosis, this could explain some of the association seen. The fact that the effect of adherence is as strong in the placebo group as in the groups receiving active treatment has given rise to the term "the healthy adherer effect", describing adherence to drug therapy as a surrogate marker for overall healthy behaviour. ${ }^{13-15}$ We did not find any difference in smoking status at baseline between patients adherent or not adherent to treatment. However, patients adherent to study medication could have better adherence to pulmonary rehabilitation and physical activity in general, which are both associated with better survival. ${ }^{16}{ }^{17}$ Unfortunately, we do not have data on these variables in the TORCH study. However, given the striking effect of adherence, small differences in physical activity are unlikely to explain our findings. Our study also suffers from lack of data on adherence over the course of the study period. Such data would have allowed more detailed statistical analyses; however, given the robust findings in this study, we doubt that it would have changed the overall conclusion. In addition, there was differential drop-out in the TORCH study. This has affected the study outcomes, but it is not clear how this may affect any association between

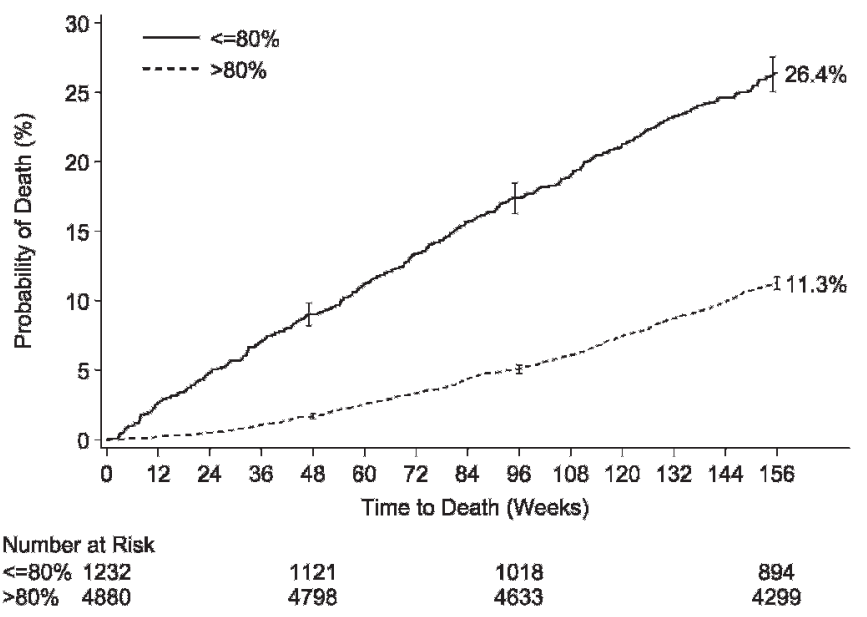

Figure 1 Kaplan-Meier plot of survival in patients adherent to study treatment and patients not adherent. 
Table 2 Causes of death among patients randomised to treatment with salmeterol (SAL), fluticasone propionate (FP), salmeterol/fluticasone propionate combination (SFC) or placebo according to degree of adherence

\begin{tabular}{|c|c|c|c|c|c|c|c|c|}
\hline & \multicolumn{2}{|c|}{ SAL (N = 1521) } & \multicolumn{2}{|c|}{$\mathrm{FP}(\mathrm{N}=1534)$} & \multicolumn{2}{|c|}{ SFC (N = 1533) } & \multicolumn{2}{|c|}{ Placebo (N = 1524) } \\
\hline & Poor & Good & Poor & Good & Poor & Good & Poor & Good \\
\hline N & 294 & 1227 & 303 & 1231 & 309 & 1224 & 326 & 1198 \\
\hline COPD, n (\%) & $20(7)$ & $44(4)$ & $18(6)$ & $49(4)$ & $19(6)$ & $24(2)$ & $19(6)$ & $41(3)$ \\
\hline COPD-related deaths, $\mathrm{n}(\%)$ & $32(11)$ & $61(5)$ & $31(10)$ & $75(6)$ & $32(10)$ & $40(3)$ & $31(10)$ & $60(5)$ \\
\hline Cardiovascular, n (\%) & $20(7)$ & $25(2)$ & 27 (9) & $34(3)$ & $24(8)$ & $36(3)$ & $25(8)$ & $46(4)$ \\
\hline Pulmonary, n (\%) & $27(9)$ & $53(4)$ & $25(8)$ & $66(5)$ & $29(9)$ & $32(3)$ & $25(8)$ & $49(4)$ \\
\hline Cancer, n (\%) & $11(4)$ & $33(3)$ & $19(6)$ & $32(3)$ & $10(3)$ & $34(3)$ & $16(5)$ & $29(2)$ \\
\hline Unknown, n (\%) & $8(3)$ & $6(<1)$ & $9(3)$ & $4(<1)$ & $10(3)$ & $7(<1)$ & $11(3)$ & $7(<1)$ \\
\hline
\end{tabular}

COPD, chronic obstructive pulmonary disease.

adherence and prognosis. Patients withdrawing from trials generally have a poorer prognosis than those remaining in a trial and, if sicker patients were more likely to have poor adherence, this could potentially explain why the association between adherence and prognosis was as strong in the placebo group as in those receiving active treatment. However, this is speculative and not supported by the data shown in table 1 .

This study is an observational study nested in a randomised controlled trial, and the patients have indeed not been randomly allocated to be good and poor adherers. The associations found are therefore likely to reflect the factors determining adherence, including health beliefs, personality, education, etc. We do not have information on these factors, and future research needs to address these. We can say, however, that the association is not a result of differences in the usual markers of disease severity, smoking habits or simple demographic descriptors. The fact that adherence is a marker of the individual patient's general behaviour and personality, and reflects more than just the use of prescribed drugs, should not be a deterrent to using the data when prescribing medication. Controlled trials have pointed to interventions such as involving pharmacies more and using telephone reminders to improve adherence and hence disease outcomes. ${ }^{18}$ Indeed, non-adherence should be seen as a challenge and not a sign of lack of cooperation by the patient. One way of meeting this challenge is by patient education but, to date, there is little evidence to suggest this has beneficial effects in improving COPD outcomes. Gallefoss ${ }^{19}$ and Hesselink et a ${ }^{20}$ were unable to show an effect of patient education alone on adherence to inhaled medication, and more comprehensive selfmanagement programmes including patient education have shown little effect on adherence and conflicting results on other outcomes. ${ }^{21}{ }^{22}$ It is, nevertheless, still possible that part of the "healthy adherer effect" in the TORCH study is the result of good adherers being better at recognising exacerbations early and thus treating exacerbations both quicker and better. In view of the results from the TORCH study indicating more favourable outcomes in patients with high levels of adherence, there is clearly a need for research that identifies features of non-adherence and strategies to address this. It should be kept in mind that, just as treatment of COPD needs to address the heterogeneity of the disease, any intervention focused on improving adherence to medication must take into account the diversity of patients' needs and understanding about the benefits and risks of treatment. ${ }^{23}$

Inhaled medications in COPD have symptomatic effects. Even though trial medications in our study have been shown to exert a symptomatic benefit within 2 days, ${ }^{4}$ adherence was not substantially better in the groups on active treatments compared with those on placebo. This is somewhat surprising as $65 \%$ of the patient population had severe or very severe COPD, a patient category where treatment with at least one long-acting bronchodilator is recommended and where patients with exacerbations should be considered for combination treatment. ${ }^{24}$

Adherence in the TORCH study was based on counting remaining doses in the returned inhalers. This is not optimal as data from the Lung Health Study suggested that some trial patients appear to "dump" inhaler medication before returning study medication to investigators. ${ }^{10}$ The Lung Health Study used metered dose inhalers where it may be easier to rapidly empty the canister than with powder inhalers, but we cannot preclude that a similar action may not have been taken by patients in the TORCH study. However, such action is unlikely to explain our findings and would most likely lead to an underestimation of the relationship between adherence and clinical outcomes.

In conclusion, we found a surprisingly strong association between adherence and mortality as well as risk of hospital admission due to an exacerbation in this 3-year trial of inhaled medications in patients with moderate to severe COPD. A better understanding of the factors differentiating good and poor adherence to medication, and strategies to improve adherence, could lead to beneficial interventions in COPD.

Acknowledgements: The authors thank all the patients in the TORCH study and investigators for their valuable contribution to this study.

Funding: The study was sponsored by GlaxoSmithKline. A Steering Committee comprising six academics and three representatives of the sponsor developed the original study design and concept, the plan for studying effects of adherence, approved the statistical plan, had full access to the data and was responsible for decisions with regard to publication. The study sponsor did not place any restrictions with regard to statements made in the final paper.

Competing interests: JV, PC, BC, GF, CJ and PJ have received fees from pharmaceutical companies, including GlaxoSmithKline, for speaking at meetings and participating in advisory board meetings. JV, PC, BC, GF, CJ and PJ have received support for research from pharmaceutical companies, including GlaxoSmithKline. JV's wife was an employee of GlaxoSmithKline until 2004. JA, JY, KK and LW are all GlaxoSmithKline employees and shareholders of GlaxoSmithKline.

Ethics approval: All patients gave informed consent and the study was approved by ethical review boards and conducted in accordance with the Declaration of Helsinki.

Provenance and peer review: Not commissioned; externally peer reviewed.

\section{REFERENCES}

1. Simpson SH, Eurich DT, Majumdar SR, et al. A meta-analysis of the association between adherence to drug therapy and mortality. BMJ 2006;333:15-20.

2. Rand CS. Patient adherence with COPD therapy. Eur Respir Rev 2005;14:97-101.

3. Balkrishnan R, Christensen DB. Inhaled corticosteroid use and associated outcomes in elderly patients with moderate to severe pulmonary disease. Clin Ther 2000;22:452-69.

4. Vestbo J, Pauwels R, Anderson JA, et al, on behalf of the TRISTAN Study Group. Early onset of effect of salmeterol and fluticasone propionate in chronic obstructive pulmonary disease. Thorax 2005;60:301-4. 
5. Cramer JA, Bradley-Kennedy C, Scalera A. Treatments persistence and compliance with medications for chronic obstructive pulmonary disease. Can Respir J 2007;14:25-9.

6. Vestbo J, The TORCH Study Group. The TORCH (TOwards a Revolution in COPD Health) Survival Study protocol. Eur Respir J 2004:24:206-10.

7. Calverley PMA, Anderson JA, Celli B, et al, on behalf of the TORCH investigators. Salmeterol and fluticasone propionate and survival in chronic obstructive pulmonary disease. N Engl J Med 2007;356:775-89.

8. Rand CS, Nides M, Cowles MK, et al. Metered-dose inhaler adherence in a clinical trial. Am Rev Respir Dis 1992;146:1559-64.

9. McGarvey LP, John M, Anderson JA, et al. Ascertainment of cause-specific mortality in COPD: operations of the TORCH Clinical Endpoint Committee. Thorax 2007:62:411-5.

10. Simmons MS, Nides MA, Rand CS, et al. Unpredictability of deception in compliance with physician-prescribed bronchodilator inhaler use in a clinical trial. Chest 2000;118:290-5.

11. Sin DD, Anthonisen NR, Soriano JB, et al. Mortality in COPD: role of comorbidities. Eur Respir J 2006;28:1245-57.

12. Soriano JB, Visick GT, Muellerova $\mathrm{H}$, et al. Patterns of comorbidities in newly diagnosed COPD and asthma in primary care. Chest 2005;128:2099-107.

13. Czajkowski SM, Chesney MA, Smith AW. Adherence and the placebo effect. In: Shumaker SA, Schron EB, Ockene JK, eds. The handbook of health behavior change. New York: Springer, 1990:515-34.

14. McDermott MM, Schmitt B, Wallner E. Impact of medication nonadherence on coronary heart disease outcomes. A critical review. Arch Intern Med 1997;157:1921-9.

15. Hays RD, Kravitz RL, Mazel RM, et al. The impact of patient adherence on health outcomes for patients with chronic disease in the medical outcomes study. J Behav Med 1994;17:347-60.
16. Puhan MA, Scharplatz M, Troosters $T$, et al. Respiratory rehabilitation after acute exacerbation of COPD may reduce risk for readmission and mortality - a systematic review. Respir Res 2005;6:54.

17. Garcia-Aymerich J, Lange $\mathrm{P}$, Benet $\mathrm{M}$, et al. Regular physical activity reduces hospital admission and mortality in chronic obstructive pulmonary disease: a population based cohort study. Thorax 2006;61:772-8.

18. Wu JYF, Leung WYS, Chang $S$, et al. Effectiveness of telephone counselling by a pharmacist in reducing mortality in patients receiving polypharmacy: randomised controlled trial. BMJ 2006;333:520-5

19. Gallefoss F. The effects of patient education in COPD in a 1-year follow-up randomised, controlled trial. Patient Educ Couns 2004;52:259-66.

20. Hesselink AE, Penninx BWJH, van der Windt DAWM, et al. Effectiveness of an education programme by a general practice assistant for asthma and COPD patients: results from a randomised controlled trial. Patient Educ Couns 2004;55:121-8.

21. Monninkhof E, van der Valk $\mathrm{P}$, van der Palen J, et al. Effects of a comprehensive self-management programme in patients with chronic obstructive pulmonary disease. Eur Respir J 2003;22:815-20.

22. Bourbeau J, Julien M, Maltais F, et al. Reduction of hospital utilization in patients with chronic obstructive pulmonary disease: a disease-specific self-management intervention. Arch Intern Med 2003;163:585-91.

23. Osman LM, Hyland ME. Patient needs and medication styles in COPD. Eur Respir Rev 2005;14:89-92.

24. Rabe KF, Hurd S, Anzueto A, et al. Global strategy for the diagnosis, management, and prevention of chronic obstructive pulmonary disease: GOLD Executive Summary. Am J Respir Crit Care Med 2007;176:532-55. 